pleurodesis whereby the IPC could be removed according to the presence of septations (pleurodesis in those with no septations $16 \%, 5 / 31$ vs. $13 \%, 2 / 16$ in those with septations, $\mathrm{p}=1.0$ ). There were no patients in either group in whom the drain was removed due lack of drainage in the context of a persistent pleural collection. There was no significant difference in overall complication rate according to the presence of septations (16\%, 5/ 31 , in the no septation group vs $13 \%, 2 / 16$, in the septation group, $\mathrm{p}=1.0$ ).

Conclusion These results suggest that the presence of septations on the pre-insertion thoracic ultrasound do not affect the rate of pleurodesis or drain removal due to lack of drainage and persistent pleural effusion. The numbers in the study are small and a limitation is the lack of assessment of post-procedure breathlessness in our patients (e.g. with a visual analogue scale). The presence of septations should not deter consideration of IPC insertion in the management of malignant pleural effusions.

\section{P182 PROPHYLACTIC DOXYCYCLINE FOLLOWING INDWELLING PLEURAL CATHETER INSERTION FOR MALIGNANT PLEURAL EFFUSIONS}

E Nuttall, H Balata, M Al-Aloul, M Evison, J Holme. University Hospital South Manchester

\subsection{6/thoraxjnl-2015-207770.319}

Introduction The insertion of indwelling pleural catheters (IPCs) allows outpatient based management of malignant pleural effusions. This group of patients frequently require chemotherapy and there is concern over infection rates from IPCs which has the potential to delay or prevent such treatment. The infection rate was reported as $10 \%$ in a prospective trial of IPCs. In our tertiary pleural service we have elected to routinely prescribe 7 days doxycycline $100 \mathrm{mg}$ once daily following IPC insertion. This study examined our infection rates using this practice.

Method Data was collected from a tertiary respiratory and pleural service. Prospective data is collected on all patients undergoing IPC insertion at UHSM. Immediate post-procedure data is collected and a further clinical and case-note review undertaken at 6 months. Pre-defined immediate (post-procedure), early (30 days) complications are recorded. Infection is defined as the prescription of antibiotics (oral or intravenous) for suspected or confirmed pleural infection or drain-site cellulitis. This study is a retrospective review of the prospectively maintained database. To ensure six months of follow-up data for all patients the analysis was restricted to patients undergoing IPC insertion prior to $31 / 12 / 2014$

Results 62 patients with complete datasets underwent IPC insertion between 01/01/2013 and 31/12/2014. All patients received 7 days of prophylactic doxycycline at a dose of $100 \mathrm{mg}$ OD. One patient $(1.6 \%)$ suffered drain site cellulitis requiring antibiotics within 30 days of insertion. There were two cases (3.2\%) of pleural infection treated with antibiotics (both within 30 days of insertion and required intravenous antibiotics and admission). In these cases the IPCs were not removed but it did fall out in one case where the patient developed delirium with infection.

Conclusion The infection rate in this prospectively collected data is lower than rates reported in large prospective randomised controlled clinical trials. This may suggest a benefit from the routine use of prophylactic antibiotics. A randomised controlled trial of prophylactic antibiotics versus no antibiotics following IPC insertion may be warranted.

\section{P183 MESOTHELIOMA IN RURAL SCOTLAND: A REVIEW OF 5 YEARS OF EXPERIENCE}

${ }^{1}$ JMF Thomas, ${ }^{2}$ SR Thomas. 'Aberdeen Medical School, Aberdeen, UK; ${ }^{2}$ Raigmore Hospital, Inverness, UK

\subsection{6/thoraxjnl-2015-207770.320}

Introduction Mesothelioma is often associated with areas of heavy industry. These areas generally have easy access to cardiothoracic facilities providing comprehensive services. We reviewed our experience of mesothelioma in the most rural part of Scotland to identify associated occupations and establish what effect configuration of local services, and the distance to the nearest thoracic surgical service had on clinical management.

Methods From 2010 the details of all patients in Highland with pleural mesothelioma have been recorded. The case notes of these patients were reviewed. Those who had presented and been investigated elsewhere were excluded. Information on occupation, investigations selected, treatment given, and survival were recorded.

Results We identified 47 mesothelioma patients. Of these 10 patients were excluded because of non-availability of notes, peritoneal mesothelioma, or presentation and investigation elsewhere. Of the remaining 37 cases the mean age at diagnosis was 71 years, $86 \%$ were male, and occupations included electricians, engineers, sheet metal workers, plumbers, joiners, builders, and shipyard workers. Two patients had been exposed in the nuclear industry. Fourteen patients underwent Abram's pleural biopsy, of which 2 (14\%) were diagnostic, 18 patients had CT guided biopsy of which $12(67 \%)$ were diagnostic, and 13 patients had thorascopic biopsy of which 10 (77\%) were diagnostic. Median survival was 10.9 months (interquartile range 12.6 months). Those with performance status (PS) 0 had median survival of 31.7 months, and PS $1,8.8$ months ( $<<0.001$ PS 0 Vs PS 1 ). For patients PS $0-2$ who received at least 1 cycle of chemotherapy median survival was 12.9 months compared with 5 months without. All were discussed in MDT meetings. 25 patients had identifiable asbestos exposure, but only in 9 of these (36\%) was there clear documentation that asbestos compensation advice had been given.

Conclusions The Scottish Highlands is affected by mesothelioma despite the rural setting. Abram's pleural biopsy is not an effective technique for suspected mesothelioma and is not appropriate even if access to thorascopic biopsy involves considerable travel. The prognosis remains poor, and although survival is better in patients who receive chemotherapy, this could be explained by pre-existing differences in those selected for chemotherapy.

\section{P184 MODIFIED WHO SAFETY CHECKLIST FOR PLEURAL INTERVENTIONS - PREVENTING SYSTEM ERRORS}

N Hutchinson, K Marshall, H Espley, AA Ionescu. Aneurin Bevan University Health Board, Newport, UK

\subsection{6/thoraxjnl-2015-207770.321}

Introduction Pleural interventions (PI) are generally safe, however adverse events occur due to human error or system failure. The World Health Organisation (WHO) safety checklists are widely used in surgery and reduce complications. ${ }^{1}$ The Royal College of Physicians has recently advocated their use for invasive medical procedures. ${ }^{2}$ 